\title{
IMPLEMENTATION OF MAGNETIC MEASUREMENTS, ERRORS, AND THEIR CONSEQUENCE IN AN OPTIMIZED FERMILAB 8-GEV TRANSFER LINE ${ }^{\dagger}$
}

\author{
S. Assadi, J. Johnstone, P. Martin, F. Tecker, M. J. Yang, Fermi National Accelerator Laboratory, \\ Batavia, IL.
}

\section{Abstract}

The purpose of the newly constructed $8 \mathrm{GeV}$ transfer line is to provide a reliable interconnection of the Booster and the Main Injector. This transfer line is constructed from the combination of the Permanent and Electro-magnets. We present data obtained during the commissioning along with our experience of combined function permanent magnets , DC operation of the electro-magnets and optics model vs. measurement of the beamline is also presented.

\section{INTRODUCTION}

The new $8 \mathrm{GeV}$ line differs in concepts from a conventional transfer lines implemented at Fermilab. The line consists of two matching sections, one to Booster and the other to the Main Injector are built from tunable electromagnet quadrupoles. However, the long repetitive FODO cells are made from permanent dipoles, gradient magnets, and quadrupoles [Fig.1].

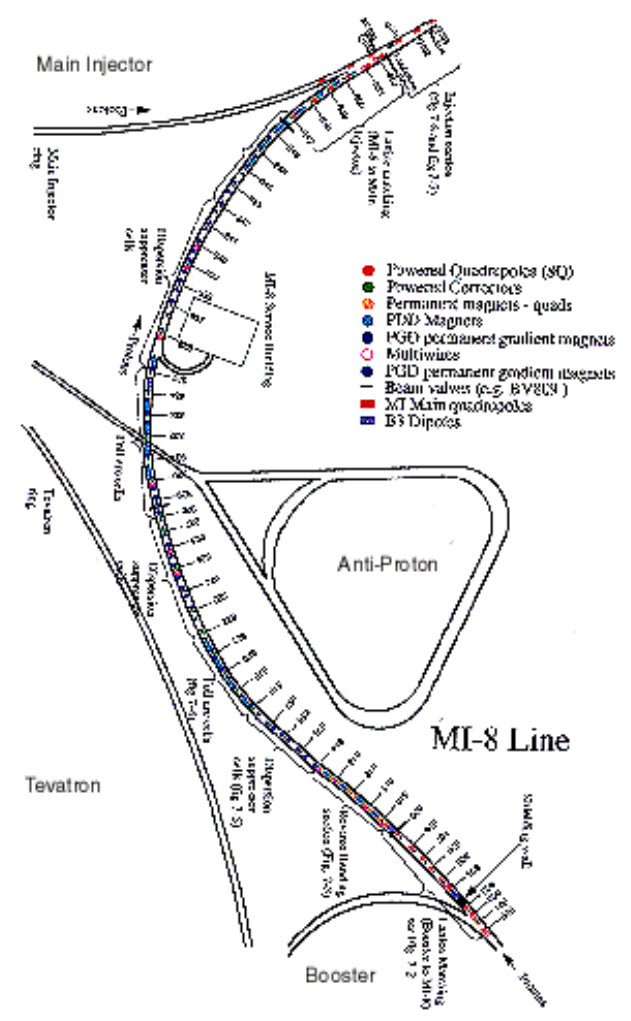

†Work supported by the US Department of Energy under contract DE-AC02-76CH00300.
The layout of the beamline and site coordinate with respect to other accelerators are shown. The vertical extraction from Booster is facilitated by a pulsed septum in the straight section L3 with an angle of 2.77 degrees. In this paper, the origin is the middle of the L3. A vertical dipole (VBC1) levels the trajectory $~ .50$ meter above the Booster elevation followed by $6.54^{\circ}$ horizontal bend. There are 2 vertical doglegs afterward to bring the beam to the Booster elevation without impacting civil constructions.

The motivation for employing permanent magnets in the line was to acquire the manufacturing and operation experience necessary to ensure the success of the $8 \mathrm{GeV}$ Recycling Ring. Important questions such as the temperature dependence, field quality and exposure to radiation of the permanent magnets resulted in commissioning and operation of the first section (station M833). Using the $\mathrm{C}++$ online model, we learned that 9 PQP's in the reversed bend section from M810-M814 were weaker by $\sim 15 \%$ that were replaced promptly. We also concluded that permanent vertical bend magnets at the beginning of the line need to be replaced due to aperture limitations and correctors needed to control the orbit throughout the beamline.

\section{MEASUREMENTS AND ANALYSIS}

There are over 50 Beam Position Monitors (BPMs), 40 Beam Loss Monitor (BLMs), 15 multi-wires and 2 toroids to measure the intensities at the entrance and exit of the transfer line. Fermilab control system provides magnetic currents, the power supply readbacks, distributed tunnel temperature and so on.

We first calibrated the quadrupole transfer constant by using 3-bump measurements. Fig. 2 presents the closure of 3-bump Q808-Q810-Q812 constructed using dipole trims. The closure confirms the power supply calibration and it is a useful tool for calibrating the quadrupole fields. The trajectory closure is sensitive, to first order only to the middle quadrupole's field as the phase advance is the same between the quads. Three-bumps are also used to center the beam through the quadrupoles and dynamic aperture is measured. The Booster matching section has ten SQ series quadrupoles. SQ's were originally designed for the Antiproton Source.

Lattice functions for the $8 \mathrm{GeV}$ line is measured by single 
kick method. A reference nominal beam data is subtracted from a one-bumped orbit. Results are compared with the model. In Fig. 3, horizontal and vertical measured BPM orbit is compared with the model.

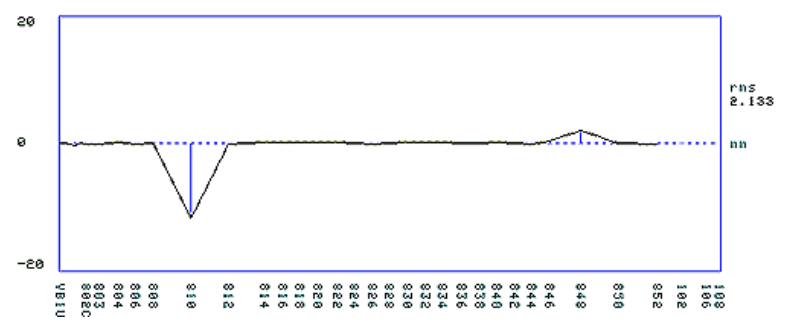

Fig. 2) The closure of 3-bumps confirms the PS calibration and the middle quadrupole field.

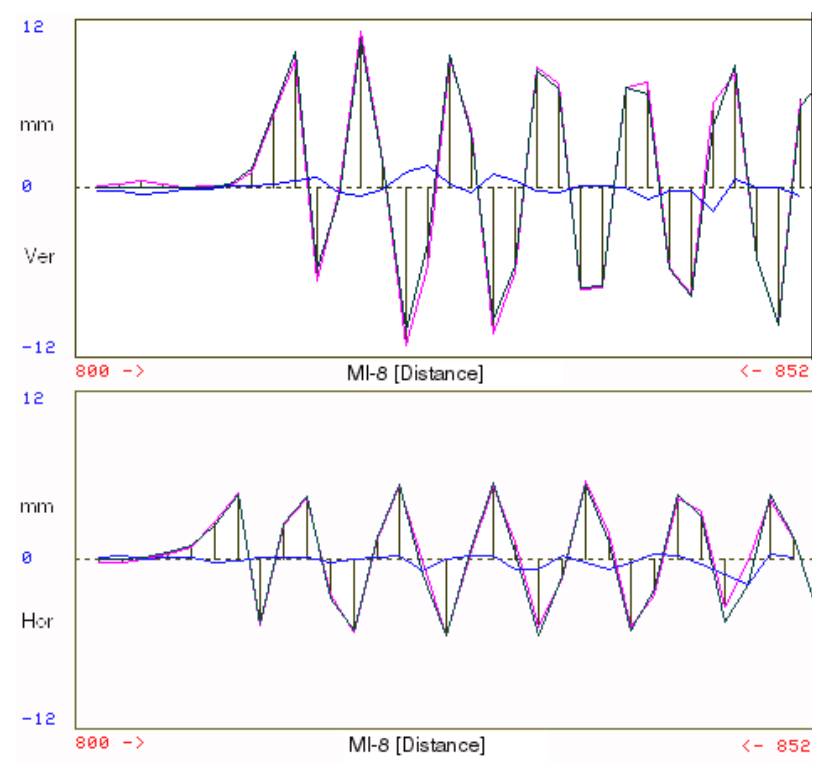

Fig.3) Vertical (Top), Horizontal (bottom) BPM difference orbit shown. Green line is the measurement, red is produced by the $\mathrm{C}++$ online model and the error is shown in blue.

We should point out that the magnitude of the error in the horizontal plane has systematically increased. This error which is proportional to the phase advance error is partly due to the alignment of the combined permanent function magnets, partly due to running slight mismatch that initially were not detected due to BPM resolution during 3-bump quad field calibration.

Due to economic reasons, a number of decisions had to be made during the construction of the MI-8. We shared the power supplies among the electromagnets. Table 1 Lists the arrangements. We also cut cost by operating the electromagnets as DC powered devices. We have been concerned about shared power supplies among the quads coupled by shunts and DC operation of the quads. Fig. 4 shows the operation of the phase one of the MI-8 line.

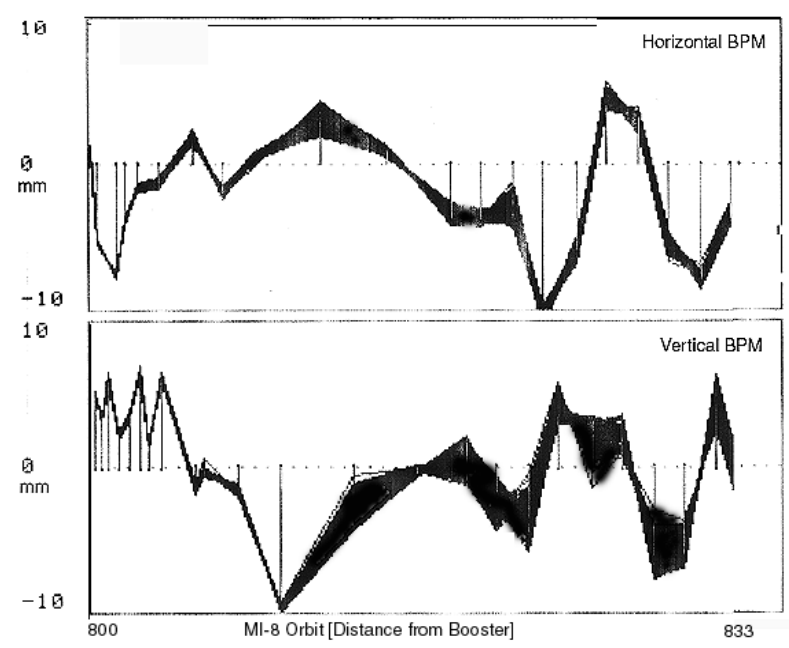

Fi.4) over-plot of 120 consecutive BPM orbits show substantial orbit deviation as beam propagates toward the Main Injector. The injection from the Booster is shown to be stable.

Reproducibility, high efficiency and emittance preservation has been our top priority during the commissioning of the MI- 8 beam line. Understanding the orbit variation shown above is the subject of the remainder of this paper.

\begin{tabular}{|l|l|}
\hline Q800-Q804-Q805 & One power supply \\
\hline Q801-Q808-Q809 & One power supply \\
\hline Q802-Q806-Q807 & One power supply \\
\hline Q803 & One power supply \\
\hline
\end{tabular}

Table 1. Shows the arrangement of the PS and shunts.

We had previously observed that the Beamline orbit could change dramatically as temporarily one of the shunt currents were varied and brought back to the nominal value. The anomalous latency effect could last for minutes although the current readbacks were returned to normal. We performed a systematic study of Q809. The quadrupole current is drived off a bulk supply minus the shunt circuit current. To enhance the effect from Q809, a positive or a negative 3-bumps with and without the large shunt setting change around the Q809 was set up. After the shunt setting was restored to its nominal value, we recorded the orbit at approximately one minute interval. Table 2. Shows the conditions and Figure 5 presents the results. We analyzed the data for the change in Q809 current required to close the 3-bump orbit ( the 3-bumps using trims VT807,VT809, and VT811 was originally closed). All trim magnet reading and settings had 
previously calibrated and verified to be reproducible.

\begin{tabular}{|l|l|}
\hline Frame 1 to 3 & Nominal setting \\
\hline Frame 4 \& 5 & Negative 3-bump \\
\hline Frame 6 \& 7 & Positive 3-bump \\
\hline Frame 8 \& 9 & $\begin{array}{l}\text { +3-bump + 46 Amps on } \\
\text { shunt (reduced quad } \\
\text { current) }\end{array}$ \\
\hline Frame $10 \& 11$ & Negative 3-bump \\
\hline Frame 12 to 27 & $\begin{array}{l}\text { Shunt is set back to } \\
\text { nominal value. }\end{array}$ \\
\hline
\end{tabular}

Table 2 presents the quad 809 shunt current setting condition. Figure five shows that the orbit variation is independent of the sign of the three bump through the

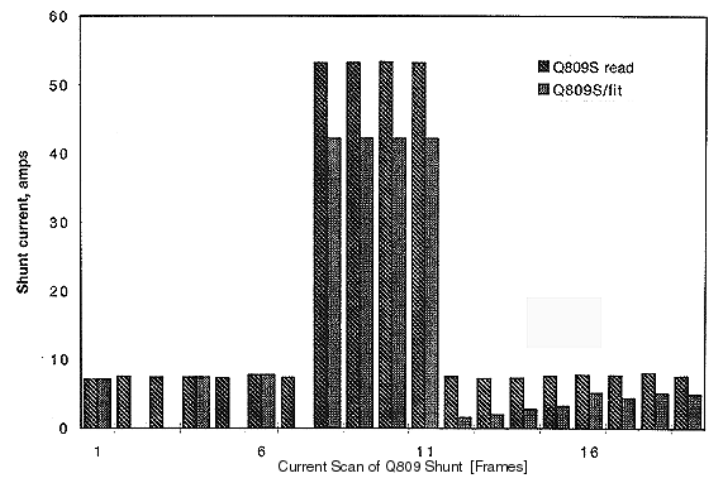

Fig.5) The calculated shunt current from frame 12 on are consistent with observed orbit changes over time.

Q809 and the reported shunt current is incompatible with the actual Q809 field. The shunt current regulation is under investigation.

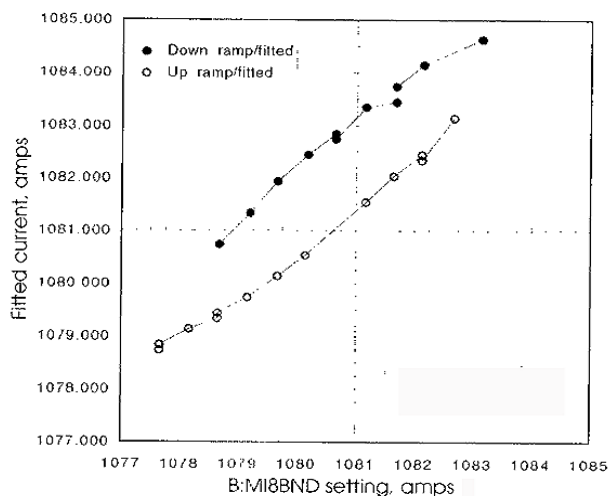

Fig. 6) The magnetic hysterisis effect is known.
Another restriction on the operational reproducibility of the MI- 8 line is the DC operation of the powered magnets and the turn-on procedure. Figure 6 shows the well known magnetic hysterisis effect of the Bend magnet. Figure 6 shows the bulk power supply MI8BND which powers four magnets has to have a designated turn-on procedure. By not accounting the hysterisis effect, the error could be as large as 2 -amp error or $12 \mathrm{~mm}$ orbit deviation at the Main Injector entrance.

We have also established the underlying reason correlating the orbit variation are related to leaking RF

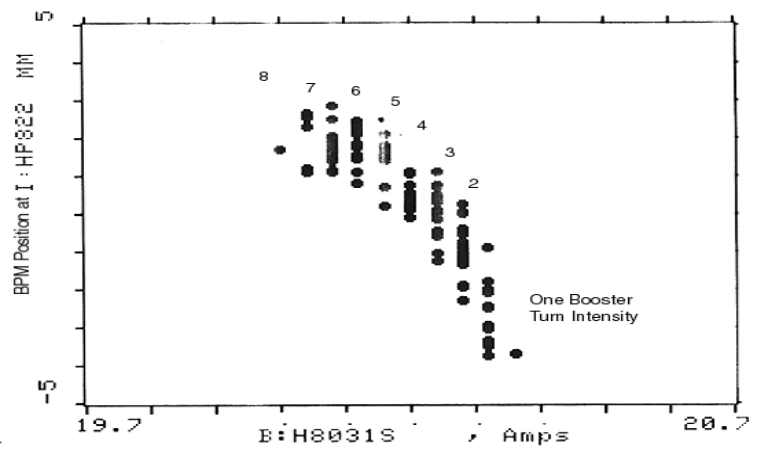

Fig.7) Beam position variation with intensity is due to shunts current being affected.

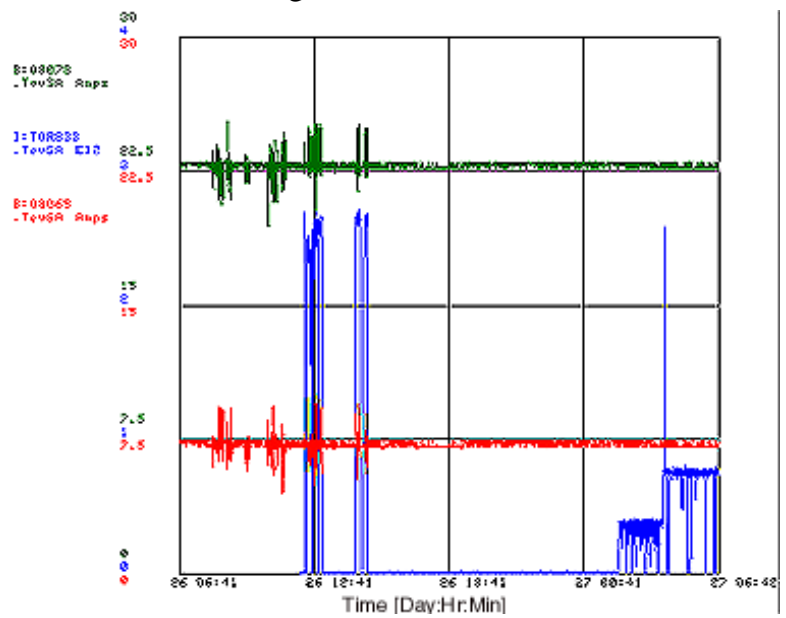

Fig. 8) During high intensity operations, shunts are affected and results in the observed orbit variation.

from the Booster affecting the shunts electronics.

\section{CONCLUSION}

We have an excellent understanding of the MI-8 optics and the permanent magnet section of the beamline has performed per design. Orbit variation is well correlated to be due to RF from the Booster affecting the electronics and are being repaired. Turn-on procedure is essential for the reproducibility and $\sim 97 \%$ beam transfer efficiency is achieved. 